Modern Physics Letters B

(C) World Scientific Publishing Company

\title{
Local Entanglement Entropy at the Mott Metal-Insulator Transition in Infinite Dimensions
}

\author{
Dan-Dan Su \\ Department of Physics, Renmin University of China, \\ Beijing 100872, P. R. China \\ Xi Dai \\ Beijing National Laboratory for Condensed Matter Physics, Institute of Physics, \\ Chinese Academy of Sciences, Beijing 100190, China \\ Ning-Hua Tong \\ Department of Physics, Renmin University of China, \\ Beijing 100872, P. R. China \\ nhtong@ruc.edu.cn \\ Received (Day Month Year) \\ Revised (Day Month Year)
}

\begin{abstract}
We study the critical behavior of the single-site entanglement entropy $S$ at the Mott metal-insulator transition in infinite-dimensional Hubbard model. For this model, the entanglement between a single site and rest of the lattice can be evaluated exactly, using the dynamical mean-field theory (DMFT). Both the numerical solution using exact diagonalization and the analytical one using two-site DMFT gives $S-S_{c} \propto$ $\alpha \log _{2}\left[\left(1 / 2-D_{c}\right) / D_{c}\right]\left(U-U_{c}\right)$, with $D_{c}$ the double occupancy at $U_{c}$ and $\alpha<0$ being different on two sides of the transition.
\end{abstract}

Keywords: Hubbard model; Metal-insulator transitions; Entanglement entropy.

\section{Introduction}

The concept of quantum entanglement plays a key role in the field of quantum information manipulation and processing 1 . It describes the inseparability between parts of a given system in a given state. In the past decade, the close relation between quantum entanglement and the quantum correlation in many-body systems are discussed $\frac{2}{2}$, especially in the context of condensed matter physics 3 , cold atoms $\frac{4}{4}$, and quantum chemistry $\underline{5}$, etc.

One of the most interesting ideas is to employ the entanglement entropy as an indicator of quantum phase transition $(\mathrm{QPT}){ }^{6}$ in both spin systems $\left.7 / 8|9| 10|11| 12\right] 13$ and the interacting fermion systems $14 \mid 15] 16|17| 18|19| 20$. For those quantum phase transitions that cannot be described by Landau's symmetry breaking paradigm, there is no well defined local order parameters. In such cases, the entanglement 
entropy may be a really useful concept for characterizing the quantum phase.

One of such QPT is the Mott metal-insulator transition, where a many-body system transits from a metallic state into an insulator, when the interaction strength between particles exceeds a critical value 21 . Experimentally, Mott transition (MT) has been widely studied both in strongly correlated electron systems such as $\mathrm{V}_{2} \mathrm{O}_{3} 22$ and in cold atom systems 23 . In the most strict sense of the Mott transition, no symmetry breaking occurs at the transition and hence it belongs to the type outside the Landau's paradigm. Since 1960's, Mott transition has been one of the key issues in condensed matter physics. Theoretically, intensive studies based on the Hubbard-type models have been done in the past decades 24 . The study of MT in terms of the quantum entanglement, especially using the entanglement entropy as a measure, appears for one-dimensional $16|18| 1920$, two-dimensional 15 , and infinite dimensional ${ }^{25}$ Hubbard-like models.

For fermionic lattice models, a connection has been established rigorously between the singularity of single-site entanglement entropy $S$ and the order of QPT, under certain conditions 19 . This connection states that the discontinuity in the (k - 1)-th order derivative of S gives a k-th order QPT. For one dimensional Hubbard model, $S$ reaches a maximum at $U_{c}=0$ where MT occurs 5 , due to equal population of all the local bases at the transition point 17 . In two dimensions, study on finite size system does not disclose any singularity at $U_{c}^{15}$. In this paper, we focus on the single-site entanglement entropy $S$ near the MT in the fermionic Hubbard model in infinite spatial dimensions. In this limit, the spatial fluctuations of electrons are suppressed while the local quantum fluctuation remains. The Hubbard model can be solved exactly in this limit using the dynamical mean-field theory $(\mathrm{DMFT}) 26$ 26 Recently, DMFT is used to evaluate the relative entropy 28 as a measure of correlation for the Hubbard model as well as for a series of transition metal oxides 25 . For the half-filled Hubbard model, at low temperature, the Fermiliquid state in small $U$ regime is separated from the Mott insulator state in large $U$ regime by a special second-order QPT27/29/30. Although this Mott transition in large spatial dimensions has received considerable attention in the past years, no analysis has been carried out for the critical behavior of its entanglement properties. We expect that such analysis can help to deepen our understanding of the MT in general.

The structure of this paper is as follows. In Section 2, we introduce the singlesite entanglement entropy $S$ and the method that we used to evaluate it for the Hubbard model, i.e., the DMFT with exact diagonalization and the two-site DMFT. In Section 3, we present numerical as well as analytical results for $S$ near the MT. In Section 4 we end with a brief summary. 


\section{Model and Method}

The Hamiltonian of the Hubbard model reads $(\hbar=1)$

$$
H=-\sum_{i, j} t_{i j} c_{i}^{\dagger} c_{j}+U \sum_{i} n_{i \uparrow} n_{i \downarrow}-\mu \sum_{i \sigma} n_{i \sigma} .
$$

Here, $t_{i j}$ is the hopping matrix element and $U$ is the on-site repulsion of electrons with opposite spin. $c_{i \sigma}$ and $c_{i \sigma}^{\dagger}$ are annihilation and creation operators of the electron on site $i$ with spin $\sigma$, respectively. $\mu$ is the chemical potential.

To study the local entanglement entropy, we divide the whole lattice into two parts, subsystem (A) (a single site $i$ ) and the environment (B) (the rest part of the lattice). For a given quantum state $|\psi\rangle$ of the whole system, the reduced density matrix of the subsystem is

$$
\hat{\rho}_{\mathrm{A}}=\operatorname{Tr}_{\mathrm{B}}|\psi\rangle\langle\psi| .
$$

The bipartite entanglement entropy between the subsystem and the environment is defined as (setting $k=1$ )

$$
S=-\operatorname{Tr}\left(\hat{\rho}_{\mathrm{A}} \log _{2} \hat{\rho}_{\mathrm{A}}\right)=-\operatorname{Tr}\left(\hat{\rho}_{\mathrm{B}} \log _{2} \hat{\rho}_{\mathrm{B}}\right) .
$$

From Eq.(2), one gets $\left\langle\psi\left|O_{A}\right| \psi\right\rangle=\operatorname{Tr}\left(\rho_{A} O_{A}\right)$ for any given operator $O_{A}$ of the system. Taking $O_{i}=1, n_{i \uparrow}, n_{i \downarrow}$, and $n_{i \uparrow} n_{i \downarrow}$ for site $i$, one gets 4 equations about the diagonal elements of $\rho_{i}$ under the basis set $(|0\rangle,|\uparrow\rangle,|\downarrow\rangle,|\uparrow \downarrow\rangle)$. Here we study the symmetry unbroken ground state of Hubbard model $|\psi\rangle$. The off-diagonal elements are all zero due to the $U(1)$ and $S U(2)$ symmetries of Hubbard model, i.e., $\left\langle\psi\left|c_{i \sigma}^{\dagger}\right| \psi\right\rangle=\left\langle\psi\left|c_{i \uparrow}^{\dagger} c_{i \downarrow}^{\dagger}\right| \psi\right\rangle=\left\langle\psi\left|c_{i \uparrow}^{\dagger} c_{i \downarrow}\right| \psi\right\rangle=0$. We therefore obtain

$$
\rho_{\mathrm{A}}=\left(\begin{array}{cccc}
\left\langle\left(1-n_{\uparrow}\right)\left(1-n_{\downarrow}\right)\right\rangle & 0 & 0 & 0 \\
0 & \left\langle n_{\uparrow}\left(1-n_{\downarrow}\right)\right\rangle & 0 & 0 \\
0 & 0 & \left\langle\left(1-n_{\uparrow}\right) n_{\downarrow}\right\rangle & 0 \\
0 & 0 & 0 & \left\langle n_{\uparrow} n_{\downarrow}\right\rangle
\end{array}\right) .
$$

The averages are with respect to the ground state of Hubbard model, and translation invariance is assumed here. For a half-filled lattice in the paramagnetic phase where the Mott transition occurs, one has $\left\langle n_{\uparrow}\right\rangle=\left\langle n_{\downarrow}\right\rangle=1 / 2$. The entanglement entropy thus reads 14

$$
S=-2\left(\frac{1}{2}-D\right) \log _{2}\left(\frac{1}{2}-D\right)-2 D \log _{2} D .
$$

Here $D \equiv\left\langle n_{\uparrow} n_{\downarrow}\right\rangle$ is the expectation value of the double occupancy. For the the ground state of the Hubbard model in infinite spatial dimensions, this quantity can be readily evaluated from the converged self-consistent solution of DMFT.

The DMFT is a well-developed theory for treating the Hubbard-type strongly correlated models 27 . In DMFT, the Hubbard model is first mapped into an effective Anderson impurity model,

$$
H_{i m p}=\sum_{k \sigma}\left[\epsilon_{k} a_{k \sigma}^{\dagger} a_{k \sigma}+V_{k}\left(a_{k \sigma}^{\dagger} c_{\sigma}+c_{\sigma}^{\dagger} a_{k \sigma}\right)\right]+U c_{\uparrow}^{\dagger} c_{\uparrow} c_{\downarrow}^{\dagger} c_{\downarrow}-\mu \sum_{\sigma} c_{\sigma}^{\dagger} c_{\sigma} .
$$


Here $a_{k \sigma}$ is the annihilation operator of bath site $k$, and the the parameters of the electron bath $\left\{\epsilon_{k}, V_{k}\right\}$ determine the dynamical "Weiss field"

$$
\mathcal{G}_{0}^{-1}\left(i \omega_{n}\right)=i \omega_{n}+\mu-\sum_{k} \frac{V_{k}^{2}}{i \omega_{n}-\epsilon_{k}} .
$$

The impurity model is then solved to generate the impurity Green's function $G\left(i \omega_{n}\right)$ on the Matsubara frequency axis. Finally, through the self-consistent equation

$$
G\left(i \omega_{n}\right)=\int_{-\infty}^{\infty} \frac{\rho_{0}(\epsilon)}{i \omega_{n}+\mu-\epsilon-\Sigma\left(i \omega_{n}\right)} d \epsilon
$$

together with $\Sigma\left(i \omega_{n}\right)=\mathcal{G}_{0}^{-1}\left(i \omega_{n}\right)-G^{-1}\left(i \omega_{n}\right)$, a new "Weiss field" $\mathcal{G}_{0}^{-1}\left(i \omega_{n}\right)$ can be obtained and used to update the bath parameters $\left\{\epsilon_{k}, V_{k}\right\}$. This process iterates and the converged solution of the impurity self-energy will be taken as the local self-energy of the lattice model, i.e., $\Sigma_{i j}\left(i \omega_{n}\right)=\Sigma_{i m p}\left(i \omega_{n}\right) \delta_{i j}$.

We adopt the semi-circular density of states

$$
\rho_{0}(\epsilon)=\frac{2}{\pi W^{2}} \sqrt{W^{2}-\epsilon^{2}}
$$

$W=1$ is set as the energy unit. Eq.(9) is the density of states of free electrons on the Bethe lattice with infinite coordination number. It is widely used in the study of the MT because it simplifies the self-consistent equation while keeps the qualitative physics intact. We first solve DMFT equations using the exact diagonalization (ED) method of Caferral et al. 31 . Then, we resort to the two-site DMFT 32 for analytical results. At half filling, this theory is reduced to the linearized DMFT 33 . It is shown that it can produce rather accurate $U_{c}$ as well as physical quantities near the critical point.

In the following, we present our results for $D$ and $S$ as a function of $U$, both from DMFT with ED and from the analytical two-site DMFT formulism.

\section{Results and Discussions}

\subsection{Exact diagonalization results}

It has been known $27 / 30$ that for finite temperatures $0<T<T_{c}$, the metallic state for small $U$ is separated from the insulating-like state for large $U$ by a finite regime $U_{c 1}(T)<U<U_{c 2}(T)$, in which the metal and the insulator phases coexist. The true first order phase transition occurs at $U_{c}(T)\left(U_{c 1}(T)<U_{c}(T)<U_{c 2}(T)\right)$ where the free energies of the metallic and of the insulating solutions coincide. At zero temperature, $U_{c 1} \approx 2.38 W^{30}$ and $U_{c}=U_{c 2} \approx 2.94 W^{29}$. The Mott transition becomes a special second-order phase transition at $U_{c 2}(T=0): \partial E_{g} / \partial U$ is continuous but the meta-stable solution of insulator extends from large $\mathrm{U}$ regime into the regime $U_{c 1}(0)<U<U_{c 2}(0)$.

In Fig.1, we show the double occupancy $D$ and entanglement entropy $S$ as functions of $U$, obtained from DMFT with ED for a very low temperature $T=$ $10^{-4} \mathrm{~W}$, which is practically same as zero temperature. As shown in Fig.1(a), the 


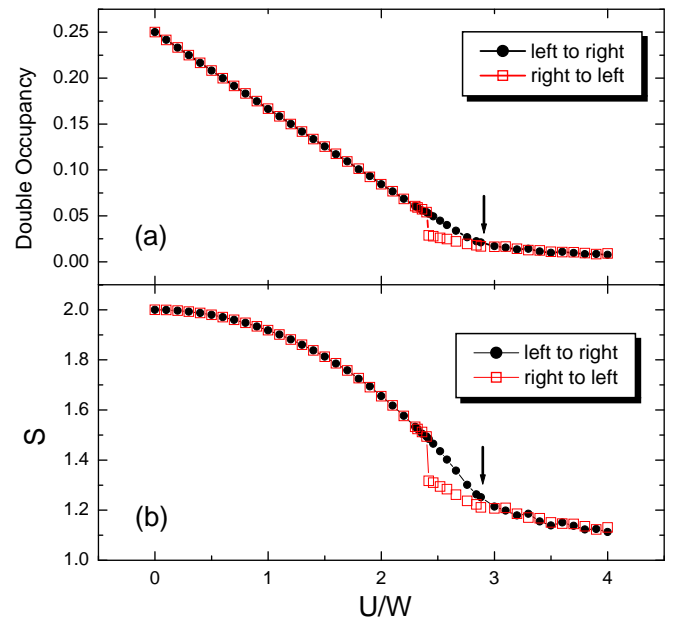

Fig. 1. (a) Double occupancy $D$ and (b) entanglement entropy $S$ as functions of $U$, obtained from ED calculation with $N_{s}=6$. Black solid dots are obtained by scanning from small $U$ to large $U$, and red empty squares are obtained by scanning from large $U$ to small $U$. Arrows mark the transition point $U_{c 2}$. Other parameters are $\mu=\mathrm{U} / 2$ and $T=10^{-4} \mathrm{~W}$.

double occupancy $D$ decreases linearly as $U$ increases up to the critical point $U_{c 2}$. Near $U_{c 2}$, coexistence of two solutions and hysteresis in $U$-scanning are observed. From the solution of $D$ obtained by scanning from small $U$ to large $U$ (solid circles in Fig.1(a)), we can identify a linear form in the $U<U_{c 2}$ regime after a non-singular term $D_{c}$ is subtracted,

$$
D-D_{c} \propto U_{c 2}-U, \quad\left(U<U_{c 2}\right) .
$$

The other solution of $D$ (empty squares in Fig.1(a)) is obtained by scanning from large $U$ to small $U$. It extends to $U_{c 1}$ and recovers the first solution through a finite jump. From the continuation of $D$ at $U_{c 2}$ in the second solution, we can infer that in the $U>U_{c 2}$ regime, a linear behavior with a much smaller slope must hold. Therefore, we can summarize $D(U)$ as

$$
D=D_{c}+\alpha\left(U-U_{c 2}\right),
$$

with $\alpha<0$ and having different values on two sides of the phase transition. This is consistent with the scenario of the special second-order MT ${ }^{30}$ in infinite dimensions: $\partial^{2} E / \partial U^{2} \sim \partial D / \partial U$ is discontinuous. Our ED calculation gives $U_{c 1} \approx 2.4 W$ and $U_{c 2} \approx 2.9 \mathrm{~W}$, in agreement with previous results of ED and projected self-consistent technique 34 . There is a small but finite double occupancy $D_{c} \approx 0.02$ at $U=U_{c 2}$. 
This reflects that even at critical point and in the insulating state, there is residual local charge fluctuations. This has important consequence for the entanglement entropy in the insulating state. See below.

In Fig.1(b), the entanglement entropy $S$ is shown. At $U=0, S=2$ comes from the equal population of electrons on the four local states. $S$ decreases monotonously with $U$. After a inflection point at $U=U_{c 2}$, it continues to decrease towards its strong-coupling limit $S_{\infty}=1$, which comes from the spin two-fold degeneracy in the paramagnetic insulator phase. Being consistent with $D$, two solutions coexist in the regime $U_{c 1}<U<U_{c 2}$ and a hysteresis is observed. What is interesting is the nonzero critical value of the entanglement entropy $S_{c}-S_{\infty} \approx 0.24$. This reflects the residual entanglement between a single site and the others at the critical point and in the insulating phase. The critical behavior of $S$ may be easily obtained from Eq.(8) and (5):

$$
S-S_{c}=2 \alpha \log _{2}\left(\frac{1 / 2-D_{c}}{D_{c}}\right)\left(U-U_{c}\right) .
$$

Here $\alpha<0$ and it has different values on two sides of MT. Due to the finite $D_{c}$, $S$ is not singular at $U=U_{c 2}$ but its derivative is discontinuous. This is the main result of this paper.

Here is the big difference between the static mean-field theory and the DMFT. For static mean-field theories such as the Weiss mean-field theory, the entanglement will become zero as soon as the system enters the long-range ordered phase, while it stays as a constant $S=1$ in the paramagnetic phase, arising from spin two-fold degeneracy. For DMFT, although the short range spin-correlation is not taken into account, the local temporal fluctuations do generate entanglement between one site and the others. Therefore, we conclude that for the Hubbard model, the local charge fluctuations in the insulating phase is associated with a nonzero local entanglement between one site and the others.

Here we did not quest for the highest precision in our ED calculation. Near $U_{c 2}$, the critical slowing down in solving DMFT equations prevents ED from obtaining rigorous conclusion. To get a more explicit solution, we resort to the two-site DMFT, which can give analytical results in the critical regime.

\subsection{Two-site DMFT result}

In the two-site DMFT, the lattice Hamiltonian is first mapped into an Anderson impurity model with one bath site,

$$
H_{i m p}=U c_{\uparrow}^{\dagger} c_{\uparrow} c_{\downarrow}^{\dagger} c_{\downarrow}-\frac{U}{2} \sum_{\sigma} c_{\sigma}^{\dagger} c_{\sigma}+\sum_{\sigma}\left[V\left(c_{\sigma}^{\dagger} a_{\sigma}+a_{\sigma}^{\dagger} c_{\sigma}\right)+\epsilon a_{\sigma}^{\dagger} a_{\sigma}\right] .
$$

The bath parameters $\epsilon$ and $V$ are determined by 32

$$
\begin{aligned}
& n_{\text {imp }}=n_{\text {lat }}, \\
& V^{2}=z M_{2}^{(0)} .
\end{aligned}
$$


Here $n_{\text {imp }}$ and $n_{\text {lat }}$ are electron density for the impurity site and for the lattice site, respectively. $z$ is the quasi-particle weight and $M_{2}^{(0)}$ is the second order moment of the free density of states, $M_{2}^{(0)} \equiv \int_{-\infty}^{\infty} \rho_{0}(\omega) \omega^{2} d \omega$. For the semicircular density of states in Eq.(9), $M_{2}^{(0)}=W^{2} / 4$. For half-filling, the particle-hole symmetry in our model guarantees that $\epsilon=0 . V$ is to be fixed by the second equation of Eq.(14).

Eq.(13) and (14) can be solved numerically. The $n_{\text {imp }}$ and $n_{\text {lat }}$ are obtained from numerical diagonalization of $H_{i m p}$ and from the lattice Green's function, respectively. $z$ is obtained from the weight of the quasiparticle poles of the impurity Green's function. The numerical results for $D$ and $S$ are shown in Fig.2.

The two-site DMFT can also be solved analytically. For this purpose, we first solve the retarded Green's function of the impurity model at zero temperature $\left(\eta=0^{+}\right)$

$$
G_{\sigma}(\omega+i \eta)=\sum_{n}\left[\frac{\left|\left\langle G\left|c_{\sigma}^{\dagger}\right| n\right\rangle\right|^{2}}{\omega+i \eta+E_{n}-E_{g}}+\frac{\left|\left\langle n\left|c_{\sigma}^{\dagger}\right| G\right\rangle\right|^{2}}{\omega+i \eta+E_{g}-E_{n}}\right] .
$$

For the particle-hole symmetric case, the eigen values and eigen states of $H_{i m p}$ can be solved analytically. The Green's function has four poles. Two of them near $\pm \frac{1}{2} U$ are for Hubbard bands, and the other two poles near $\pm V^{2} / U$ are precursors of the coherent Kondo resonance. To calculate $z$, we take the weights of the latter two poles and expand $z$ in terms of powers of $V / U$,

$$
z=\frac{36 V^{2}}{U^{2}}-\frac{1584 V^{4}}{U^{4}}+\ldots
$$

This expression together with Eq.(14) gives the solution for $V$ as

$$
V=\frac{U}{2 \sqrt{11}} \sqrt{1-U^{2} / U_{c}^{2}} \quad\left(U<U_{c}\right),
$$

and $V=0$ for $U>U_{c}$. Here $U_{c}=6 \sqrt{M_{2}^{(0)}}$. For the Bethe lattice, $U_{c}=3 W$ is very close the the ED results $U_{c 2} \approx 2.9 W$. For $U<U_{c}, V>0$ and the system is in the metallic phase. For $U>U_{c}, V=0$ and the system is in the insulating phase. Note that this expression differs from that of Potthof ${ }^{32}$ in the prefactor, due to different ways of calculating 2 .

Exact solution of $H_{i m p}$ gives the analytical expression for the double occupancy as

$$
D_{i m p} \equiv\left\langle n_{\uparrow} n_{\downarrow}\right\rangle=\frac{\left(U-\sqrt{U^{2}+64 V^{2}}\right)^{2}}{4\left(U^{2}+64 V^{2}-U \sqrt{U^{2}+64 V^{2}}\right)} .
$$

Combining it with Eq.(17), one obtains the critical expression for $D$ as,

$$
D_{i m p}=\frac{4}{11}\left(1-U / U_{c}\right) \quad\left(U<U_{c}\right),
$$

and $D_{i m p}=0$ for $U>U_{c}$. For $S$ we have

$$
S_{i m p}=-\frac{8}{11}\left(1-\frac{U}{U_{c}}\right) \log _{2}\left(1-\frac{U}{U_{c}}\right) \quad\left(U<U_{c}\right)
$$




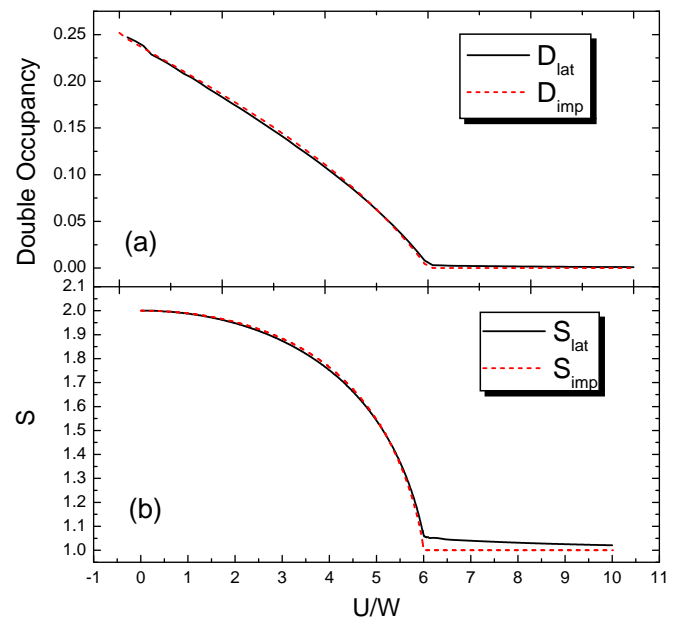

Fig. 2. Double occupancy $D$ (a) and entanglement entropy $S$ (b) as functions of $U$, obtained from numerical solution to the two-site DMFT equations. The solid lines are results for lattice model, and the dash-dot lines are for impurity model.

and $S_{i m p}=1$ for $U>U_{c}$.

However, it is noted that $S_{i m p}$ in Eq.(20) is not the correct results. Although the two-site DMFT equation requires $n_{i m p}=n_{\text {lat }}$, the double occupancy $D_{i m p}$ does not equal to its lattice counterpart $D_{\text {lat }}$, due to the fact that the two-site DMFT is an approximation to the full DMFT. In Fig.2(a), we plot $D_{i m p}$ and $D_{\text {lat }}$ as functions of $U . D_{\text {lat }}$ is calculated using the lattice Green's function 27 . It is seen that for $U<U_{c}$, both $D_{i m p}$ and $D_{l a t}$ are linear near $U_{c}$. For $U>U_{c}$, although $D_{i m p}=0$, $D_{\text {lat }}$ is small but finite, with a finite slope at $U=U_{c}$, consistent with ED results.

Correspondingly, $S_{i m p}$ calculated from $D_{i m p}$ becomes 1 immediately after $U>$ $U_{c}$, while $S_{\text {lat }}$ calculated from $D_{\text {lat }}$ is not singular at $U_{c}$. It has a linear behavior at $U_{c}$ but with a much smaller slope than that on the $U<U_{c}$ side. This agrees with the ED results. Therefore, we believe that the $S_{\text {lat }}$ is an improvement over $S_{i m p}$ which ignores the finite $D$ at $U_{c}$. This is expected because $S_{\text {lat }}$ takes into account the lattice information more elaborately than $S_{i m p}$ through the lattice Green's function. In conclusion, both the $S_{l a t}$ from two-site DMFT calculation and that of DMFT with ED give the critical behavior of the single-site entanglement entropy as described by Eq.(12).

Our conclusion Eq.(12) is exact for the Hubbard model in infinite dimensions. Being different from the one-dimensiona $\frac{16}{16}$ and two-dimensional cases $\frac{15}{\text {, our study }}$ 
shows that MT in infinite dimensions fulfills the rigorous theorem by Larsson and Johannesson, which states that the discontinuity in the $(k-1)$-th order derivative of $S$ gives a $k$-th order QPT 19 , with $k=2$ in this case. Besides the single-site entanglement entropy, two-site von Neumann entropy has been studied at the quan-

tum phase transition 17 . For Hubbard model, it can be calculated using the cluster extension of DMFT27.

\section{Summary}

To conclude, we have analyzed the critical behavior of the single-site entanglement entropy $S$ at the MT in infinite spatial dimensions, using DMFT with ED and the two-site DMFT. Even in the insulating phase, temporal local fluctuations generate nonzero $S$ besides the contribution from spin two-fold degeneracy. The critical behavior of $S$ at the MT is not singular due to finite double occupancy at $U_{c}$, but $\partial S / \partial U$ is discontinuous at $U_{c}$, being consistent with the second order scenario of MT and the Larsson-Johannesson theorem 19 .

This work is supported by National Program on Key Basic Research Project (973 Program) under grant 2012CB921704, and by the NSFC under grant number 11074302.

\section{References}

1. D. P. Di Vincenzo and C. Bennet, Nature (London) 404, 247 (2000).

2. L. Amico, R. Fazio, A. Osterloh, and V. Vedral, Rev. Mod. Phys. 80, 517 (2008).

3. P. Thunström, I. Di Marco, and O. Eriksson, arXiv:1202.3975

4. P. Buonsante 1 and A. Vezzani, Phys. Rev. Lett. 98, 110601 (2007).

5. M. Babamoradi, M. Heidari Saani, and M. A. Vesaghi, arXiv:1007.3112; N. M. Tubman and J. McMinis, arXiv:1204.4731; K. Boguslawski, P. Tecmer, Örs Legeza, and M. Reiher arXiv:1208.6586

6. S. Sachdev, Quantum Phase Transitions, Cambridge University Press, 1999.

7. T. J. Osborne and M. A. Nielsen, Phys. Rev. A 66, 032110 (2002).

8. R. G. Melko, A. B. Kallin, and M. B. Hastings, Phys. Rev. B 82, 100409(R) (2010).

9. A. B. Kallin, M. B. Hastings, R. G. Melko, and R. R. P. Singh, Phys. Rev, B 84, 165134 (2011).

10. M. B. Hastings, I. González, A. B. Kallin, and R. G. Melko, Phys. Rev. Lett. 104, 157201 (2010).

11. R. R. P. Singh, R. G. Melko, and J. Oitmaa, Phys. Rev. B 86, 075106 (2012).

12. F. Verstraete, M. A. Martín-Delgado, and J. I. Cirac, Phys. Rev. Lett. 92, 087201 (2004).

13. M. Popp, F. Verstraete, M. A. Martín-Delgado, and J. I. Cirac, Phys. Rev. A. 71, $042306(2005)$.

14. P. Zanardi, Phys. Rev. A 65, 042101 (2002).

15. J. Wang and S. Kais, Phys. Rev. A. 70, 022301 (2004).

16. S.-J. Gu, S.-Sa. Deng, Y.-Q. Li, and H.-Q. Lin, Phys. Rev. Lett. 93, 086402 (2004).

17. H. Johannesson and D. Larsson, Low Temperature Physics 33, 935 (2007).

18. D. Larsson and H. Johannesson, Phys. Rev. Lett. 95, 196406 (2005).

19. D. Larsson and H. Johannesson, Phys. Rev. A. 73, 042320 (2006).

20. W.-L. Chan and S.-J. Gu, J. Phys.: Condens. Matter 20, 345217 (2008). 
21. N. F. Mott, Proc. Phys. Soc. London, Sect. B 62, 416 (1949).

22. D. B. McWhan and J. P. Remeika, Phys. Rev. B 2, 3734 (1970); D. B. McWhan et al., ibid. 7, 1920 (1973).

23. M. Greiner, O. Mandel, T. Esslinger, T. W. Hänsch, and I. Bloch, Nature (London) 415, 39 (2002).

24. F. Gebhard, The Mott Metal-Insulator Transition, Springer-Verlag Berlin Heidelberg, 1997.

25. K. Byczuk, Jan Kunes, W. Hofstetter, and D. Vollhardt, Phys. Rev. Lett. 108, 087004 (2012); 108, 189902 (2012).

26. W. Metzner and D. Vollhardt, , Phys. Rev. Lett. 62, 324 (1989).

27. A. Georges, G. Kotliar, W. Krauth, and M. J. Rozenberg, Rev. Mod. Phys. 68, 13 (1996).

28. A. D. Gottlieb and N. J. Mauser, Int. J. Quantum. Inform. 5, 815 (2007).

29. R. Bulla, Phys. Rev. Lett. 83, 136 (1999).

30. N. H. Tong, S. Q. Shen, and F. C. Pu, Phys. Rev. B 64, 235109 (2001).

31. M. Caffarel and W. Krauth, Phys. Rev. Lett. 72, 1545 (1994).

32. M. Potthoff, Phys. Rev. B 64, 165114 (2001); J. Ortloff, M. Balzer, and M. Potthoff, Eur. Phys. J. B 58, 37 (2007).

33. R. Bulla and M. Potthoff, Eur. Phys. J. B 13, 257 (2000).

34. See Fig.34 of Ref. 27 .

35. In Ref. $\frac{32}{,} z$ is calculated via $z=1 /\left[1-\partial \Sigma(\omega) /\left.\partial \omega\right|_{\omega=0}\right]$. Here, we calculate $z$ as the weight of the qusi-particle poles in the Green's function. These two ways have slight difference away from $V=0$. 